\title{
Magnetic Resonance Perfusion in Brain Tumors: Comparison of Different Evaluation Approaches in Dual-Echo and Multi-Echo Techniques
}

\author{
Volker Hietschold1, Andrij Abramyuk2 ${ }^{2}$, Tareq Juratli3 ${ }^{3}$, Kerim Hakan Sitoci-Ficici ${ }^{3}$, \\ Michael Laniado', Jennifer Linn²
}

${ }^{1}$ Radiology, University Hospital Carl Gustav Carus, Dresden, Germany

${ }^{2}$ Neuroradiology, University Hospital Carl Gustav Carus, Dresden, Germany

${ }^{3}$ Neurosurgery, University Hospital Carl Gustav Carus, Dresden, Germany

Email: volker.hietschold@uniklinikum-dresden.de

How to cite this paper: Hietschold, V., Abramyuk, A., Juratli, T., Sitoci-Ficici, K.H., Laniado, M. and Linn, J. (2017) Magnetic Resonance Perfusion in Brain Tumors: Comparison of Different Evaluation Approaches in Dual-Echo and Multi-Echo Techniques. International Journal of Medical Physics, Clinical Engineering and Radiation Onco$\log$, 6, 174-192.

https://doi.org/10.4236/ijmpcero.2017.62016

Received: March 6, 2017

Accepted: May 20, 2017

Published: May 23, 2017

Copyright ( $\odot 2017$ by authors and Scientific Research Publishing Inc. This work is licensed under the Creative Commons Attribution International License (CC BY 4.0).

http://creativecommons.org/licenses/by/4.0/

(c) (i) Open Access

\begin{abstract}
Dynamic measurements of $T_{1}$ shortening (dynamic contrast enhanced-DCE) as well as of $T_{2}^{*}$ shortening (dynamic susceptibility contrast-DSC) as two separate measurement strategies are widely used to quantitatively describe tumor perfusion and vascularity. Dual-echo approaches allow for the simultaneous assessment of both effects. The extension to multi-echo sequences should inhere the advantage of improved signal-to-noise ratios and more precise sampling of the $T_{2}^{*}$ decay. The aim of our study is to investigate, if an extension of the dual-echo approach to the multi-echo approach allows for more stable quantitative determination of pharmacokinetic parameters in brain tumors. This study applies a multi-echo approach to obtain different estimations of a vascular input function and analyzes various combinations of vascular input functions and pharmacokinetic models. Perfusion measurements were performed with 52 consecutive patients with different brain tumors using a 10-echo gradient echo sequence. Our findings show that the extension to multi-echo sequences leads to an $11 \%$-improvement of the Contrast-to-Noise ratio. Compared to other combinations, an application of Extended Tofts model using the $T_{2}^{*}$-related venous output function or an output function estimated in the tumor tissue enables the most reliable determination of perfusion parameters, reducing the reproducibility range by a factor of 1.2 to 10 for $\mathrm{K}^{\text {trans }}$ and of 1.2 to 5.5 in the case of rBV calculation. Determination of $K^{\text {trans }}$ within repeated measurements within about 3 days results as most stable, if AIF from tumor pixels is used as vascular input function, meaning that the scatter is reduced by a factor of 1.2 compared to the next best VIF and by a factor of 10 compared to the worst of the tested approaches. In addition, this study shows that signal decomposition into two components
\end{abstract}


with different Larmor frequencies might provide additional information concerning tissue composition of brain tumors.

\section{Keywords}

MRI, Brain Tumors, Perfusion

\section{Introduction}

Dynamic contrast enhanced (DCE) magnetic resonance imaging and dynamic susceptibility contrast (DSC) magnetic resonance imaging are widely used techniques for the assessment of tumor perfusion and tumor vascularity. In DCEMRI, the measured $T_{1}$ shortening is mainly caused by contrast medium (CM) distributed in the interstitial space, but partly also by intravascular CM [1] [2]. $T_{2}^{*}$ shortening measured in DSC-MRI is predominantly determined by CM in the capillary space. However, interstitial CM accumulation has some effect on $T_{2}^{*}$ shortening as well.

Since the diffusion of CM into the interstitial space is much slower compared to the passage of a CM bolus through the capillaries, $T_{1}$-related dynamic measurements can be performed with a temporal resolution of about 45 seconds to 3 minutes [3] [4]. For this reason, spin echo or gradient echo volume sequences with short echo times (TE) can be used to minimize the influence of $T_{2}^{*}$ changes. In contrast, $T_{2}^{*}$-related measurements usually are performed with EPI sequences to cover a sufficient volume with a temporal resolution of 1 to 2 seconds [5]. Here, long repetition times (TR) assure minimal influence of $T_{1}$ shortening.

Using a dual-echo approach, temporal distribution of CM in both interstitial and capillary compartments can be assessed during only one CM administration [6]. The idea of simultaneous measurement and separation of $T_{1}$ and $T_{2}^{*}$-related signal changes was firstly proposed in the 1990s [6] [7] [8]. The basic principle of this approach is the use of a gradient dual-echo sequence in order to simultaneously obtain images with equal $T_{1}$ weighting but different $T_{2}^{*}$ weighting. The structure of the gradient echo signal intensity formula allows the calculation of the time course of $R_{2}^{*}(t)$ and at least of a hypothetical signal $S_{0}$ for $T_{E}=0$ (being independent of $R_{2}^{*}$ ).

However, to achieve a sufficient temporal resolution, this dual-echo approach is restricted to a few slices only. This major drawback might be solved by the application of more sophisticated sequence designs (e.g., parallel imaging, keyhole, segmented EPI etc.).

Another disadvantage of the dual-echo approach is the compromise between temporal resolution and signal to noise ratio (SNR) of the calculated images, which does not always produce sufficient accuracy of results.

In addition, the determination of a vascular input function (VIF) still is a critical step in MR perfusion measurements. In vessels apart from the tumor, the time course of the CM concentration is known to be different from that in the 
intratumoral vasculature in terms of temporal position (i.e., delay) as well as peak broadening (i.e., dispersion). This might result in systematic errors of estimated perfusion parameters [9].

These problems can be diminished when applying the concept of simultaneous dynamic registration of $T_{1}$ and $T_{2}^{*}$-related signals. We hypothesize that:

- The multi-echo approach results in a significant better contrast-to-noise ratio (CNR) of the calculated $R_{2}^{*}(t)$ and $S_{0}(t)$ compared to the dual-echo approach. This is because the acquisition of more than two echoes within a given TR samples the $T_{2}^{*}$ decay curve with more than two data points.

- Simultaneous dynamic $T_{1}$ and $T_{2}^{*}$-based measurement enables estimation of a $T_{2}^{*}$ based VIF from the tumor pixels in addition to the DCE perfusion properties. Thus, location related VIF bias might be avoided.

Moreover, there exists still some hidden potential of the multi-echo approach. Especially when we express the TE dependent signal intensity as a complex sum of two components with different Larmor frequencies (e.g., water and fat), we can model the signal dependence on TE more correctly with the multi-echo approach.

Therefore, the aim of this paper is to reveal the potential benefits of the multi-echo approach in dynamic imaging of brain tumors.

More precisely, we aim to assess alterations of CNR using higher numbers of echoes within a given $T_{R}$. For this purpose, different correction methods for the calculation of $S_{0}(t)$ and $R_{2}^{*}(t)$ are compared.

Additionally, different estimations of a vascular input function are compared quantitatively and various combinations of vascular input functions and pharmacokinetic models are tested.

Finally, assuming tumor tissue to consist of two components with different Larmor frequencies, we aim to obtain more information about the tumor structure by differentiating those substances. We think that at least rough spectroscopic differences are identifiable with multi-echo measurements. Therefore, we analyze the potential clinical value of the signal decomposition into two parts with different Larmor frequencies in a population of brain tumor patients.

\section{Material and Methods}

\subsection{Patients}

52 consecutive patients with different, newly diagnosed brain tumors were included in the study (25 glioblastoma, 12 meningioma, 12 metastasis, 3 lymphoma patients). For 21 patients, MRI examination was performed twice with a time interval of 1 to 4 days. 18 patients received preoperatively dexamethasone (10 glioblastoma, 2 meningeoma, 5 metastasis, 1 lymphoma patients). We excluded five patients who received an MRI examination twice and eight patients with single-time MRI examinations from further analysis due to non-plausible vascular input functions or movement artifacts.

\subsection{MRI Measurements}

MRI was performed on a 3 Tesla MR scanner (Siemens Magnetom Verio). In 
addition to the standard tumor protocol, a dynamic 10 echo FLASH sequence $\left(T_{R}=44 \mathrm{~ms}, \alpha=70^{\circ}, T_{E}=1.2,2.2,3.1,4.1,5.5,6.4,8.0,10.0,12.0\right.$ and $13.0 \mathrm{~ms}$, matrix $256 \times 208$ (acquisition matrix: $128 \times 73$ ), GRAPPA acceleration factor $=3$ ) with a temporal resolution of 2 seconds was run during the application of $\mathrm{CM}$ $(0.1 \mathrm{ml} / \mathrm{kg}$ body weight of Gadovist, (Bayer Schering) at a flow of $4 \mathrm{ml} / \mathrm{s}$ followed by $10 \mathrm{ml}$ of saline). The sequence includes 3 slices of each $5 \mathrm{~mm}$ thickness. One slice was positioned in the neck region for measurement of an Arterial Input Function (AIF), two others were placed in the tumor region, identified with native $T_{1}$ and $T_{2}$ weighted images. After 10 of 60 dynamic scans, the CM was administered.

\subsection{Multi-Echo Correction Methods (Calculation of $R_{2}^{*}$ and $S_{0}$ )}

For calculation of $R_{2}^{*}(t)$ and $S_{0}$, different procedures were applied (see appendix). The calculations were based on the first and last echo only (i.e., conventional dual-echo approach), as linear fit on the logarithmic signal intensities, as monoexponential fit on the signal intensities themselves or as fit of two complex intensities of components with different Larmor frequencies (i.e., a multi-echo Dixon approach). Calculations based on first and second echo only were also performed as negative control.

The quality of the multi-echo corrections (i.e., the determination of $S_{0}$ and $R_{2}^{*}$ maps) was evaluated by calculation of CNR ratios for the time curves of $R_{2}^{*}$ and $S_{0}$ for the AIF, VOF and tumor ROIs. For this purpose, the difference of the curve maximum and the baseline value was divided by the standard deviation along the baseline. For plausibility control, modified Euclidean distances between glioblastoma, meningioma, metastasis and lymphoma were calculated based on $\mathrm{K}^{\text {trans }}$ and $\mathrm{rBV}$ (both calculated with the $R_{2}^{*}$ VOF, see Table 1). For reasons of non-symmetric parameter distributions and noisy data, medians instead of mean values were used. Taking into account the asymmetric parameter distribution, the normalization was done with the mean difference of the 75 and 25 percentiles of the two classes being compared. As an overall quality parameter, we used the sum of these distances normalized to the size of the smaller class.

Table 1. Sum of contrast-to-noise ratios over the ROIs in tumors, arteries and venes and sum of Euclidean distances between the median values of glioblastoma, meningioma, metastases and lymphoma.

\begin{tabular}{ccc}
\hline & Sum of CNRs & Euklidean distance \\
\hline $1^{\text {st }}+2^{\text {nd }}$ echo & 163.92 & 26.6 \\
$1^{\text {st }}+10^{\text {th }}$ echo & 319.21 & 24.9 \\
10 echoes loglinear & 357.04 & 33.4 \\
10 echoes exponential & 342.41 & 33.3 \\
4 echoes exponential & 330.16 & 27.4 \\
water fat 10 echoes exponential & 347.42 & 30.4 \\
water fat 4 echoes exponential & 344.51 & 27.4 \\
\hline
\end{tabular}




\subsection{Vascular Input Function}

For estimation of the vascular input function, we implemented several time courses: $S_{0}(t)$ and $R_{2}^{*}(t)$ of a ROI in a neck artery (AIF) and in a venous sinus (mostly superior sagittal sinus-VOF) and $R_{2}^{*}(t)$ in the tumor ROI. Manual drawing of vascular ROIs was supported by pixel exclusion based on lower and upper thresholds for maximum change and time of maximum in $S_{0}(t)$ and $R_{2}^{*}(t)$ respectively. During the definition of the ROIs, both $S_{0}(t)$ and $R_{2}^{*}(t)$ were checked for plausibility control. In case of non-plausible curve shapes, other vessels or other vessel segments were selected. Baseline subtraction was performed after manual selection of a baseline range, mostly including the $4^{\text {th }}$ to $22^{\text {th }}$ scan. For the use of a $S_{0}(t)$ curve in $R_{2}^{*}(t)$ based models, the curve was normalized to the maximum of $R_{2}^{*}(t)$ and vice versa (in the case of $R_{2}^{*}$ from the tumor ROI the corresponding maxima from the healthy tissue ROI were used in order to minimize the influence of the non-linearity of the dependence of $\mathrm{S}_{0}$ from the CM concentration). As a preprocessing step, the vascular input functions were shifted in time (except for $R_{2}^{*}(t)$ in the tumor ROI). For that reason, positions of the maxima of the curves were determined by Gaussian fits to the data points exceeding $60.6 \%$ of the maximum (for normal distributions, this is equivalent to the position range of mean \pm standard deviation). The VIFs were shifted so that their maximum position became equal to that of the $R_{2}^{*}$ curve measured in the tumor ROI. When applying $R_{2}^{*}(t)$ in the tumor ROI as vascular input function, $\mathrm{rBV}$ and $\mathrm{K}^{\text {trans }}$ estimations are biased by the "real rBV" multiplicatively. Therefore, appropriate rescaling has to be done, preferable with $\mathrm{rBV}$ determined on the base of another vascular input function in order to avoid errors.

The degree of nonlinearity in $S_{0}$ (CM concentration) was qualitatively evaluated by comparing the curve shape of $S_{0}(t)$ with that of $R_{2}^{*}(t)$.

\subsection{Comparison of Pharmacokinetic Model-Vascular Input Function Combinations}

The following models were combined with the above-described vascular input functions:

For $S_{0, \text { Tumor }}(t)$ we applied the Patlak and Extended Tofts models [10] [11] [12] [13] to calculate $\mathrm{K}^{\text {trans }}, \mathrm{rBV}$ and $\mathrm{v}_{\mathrm{e}}$. The curve fits were performed with precalculated $\mathrm{rBV}$ as well as with $\mathrm{rBV}$ as free parameter.

For $R_{2 \text { Tumor }}^{*}$ we used the Indicator Dilution Theory (IDT) to determine rBV, regional blood flow (rBV) and mean transit time (MTT) [14] [15] [16]. For that, Gaussian and Gama Variate peaks were fitted to $R_{2}^{*}(t)$ curves.

Additionally, we evaluated simple empiric parameters like maximum relative and maximum absolute enhancement in $S_{0, \text { Tumor }}(t)$ and maximum temporal increase of $R_{2 \text { Tumor }}^{*}$. Each parameter was examined before as well as after normalization to the corresponding value from a ROI in contralateral healthy tissue.

Based on the perfusion parametric images, lacunarity parameters were calculated as published elsewhere [17]. 

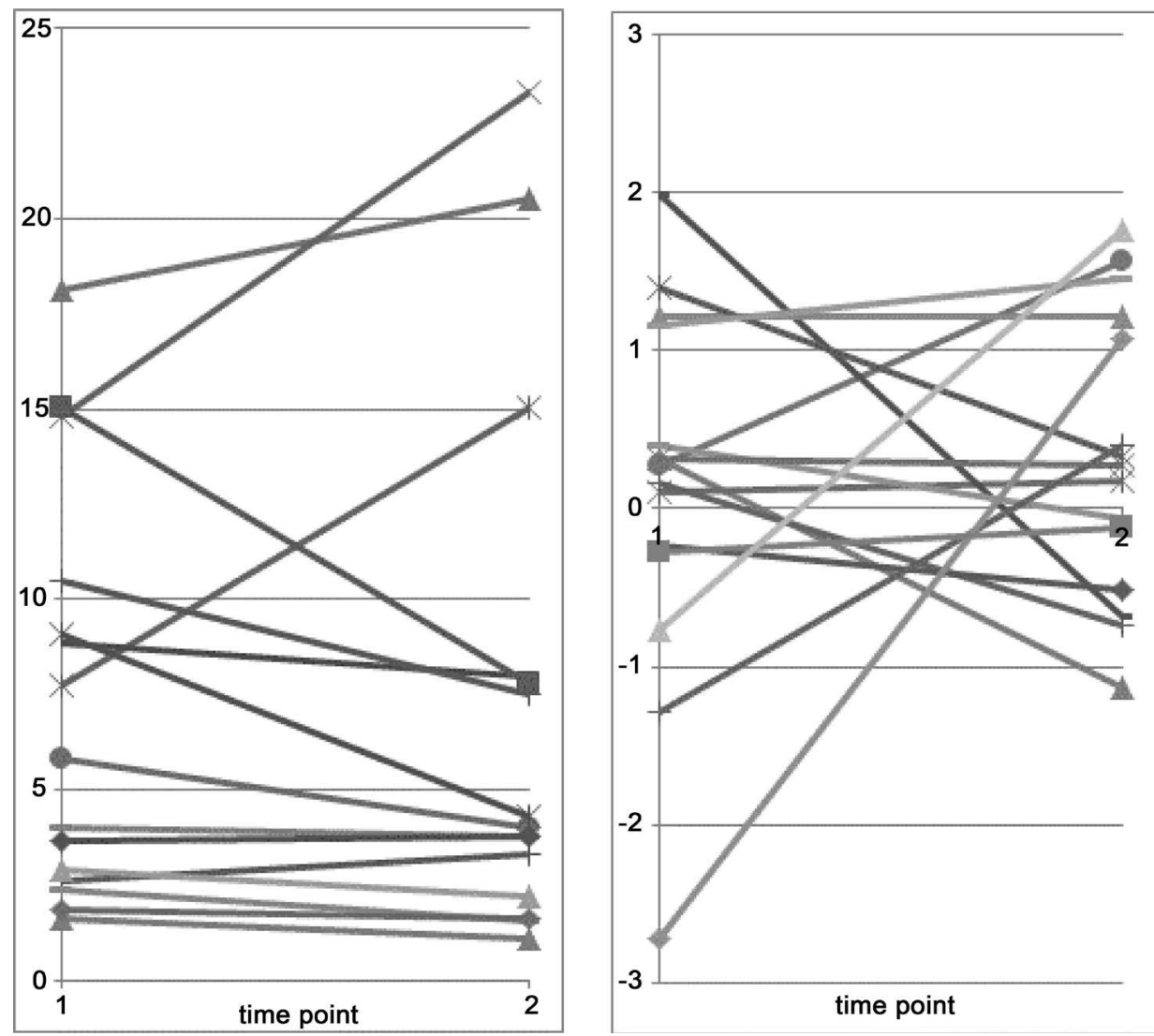

Figure 1. Examples for reproducibility diagrams: patient by patient course of parameters calculated from the first and second investigation (loglinearmultiecho correction). Left: relative enhancement of $S_{0}$ in the tumor, normalized to contralateral tissue. Good reproducibility, mean normalized deviation $\bar{\Delta}=0.62$. Right: $k_{2}$ from fit with rBV as free parameter with AIF derived from the $R_{2}^{*}$ from an artery after appropriate scaling. Bad reproducibility, $\bar{\Delta}=1.82$.

To identify model/VIF-combinations allowing the most stable perfusion parameter estimation, the reliability and reproducibility of the calculated parameters were evaluated visually and quantitatively. For the visual evaluation of each parameter, the values of the first and second investigation were drawn on a patient-by-patient basis (see Figure 1). For the quantitative assessment, a normalized mean difference between the consecutive MRI sessions was calculated to be used as reproducibility parameter

$$
\bar{\Delta}=\frac{2}{n \cdot \operatorname{median}\left(x_{1, i}+x_{2, i}\right)} \sum_{i=1}^{n}\left(x_{1, i}-x_{2, i}\right)
$$

( $n=$ number of patients, $x_{1, i}$ or $x_{2, i}=$ parameter $x$ for patient $i$ at first or second measurement resp.). This parameter normalizes the mean of difference of measures between the two time points to the median of the corresponding parameter and defines in this way a relative measure of stability of that parameter. For plausibility reasons, $\bar{\Delta}$ was used to generate a list of the 30 best normalized and 30 best non-normalized parameters, from which the most plausible ones were selected on the basis of the diagrams mentioned above. This step was necessary due to the bias resulting from a few patients with unusual large deviations be- 
tween the first and second measurements.

\subsection{Data Analysis}

Statistical analysis was done using Excel with user functions written in VBA (Visual Basic for Applications, Microsoft Corporation). The image analysis was performed with a program written in IDL ("Interactive Data Language", Exelis Visual Information Solutions Inc.).

\section{Results}

\subsection{Comparison of Multi-Echo Correction Methods (Calculations of $R_{2}^{*}$ and $S_{0}$ )}

The CNR values averaged over all measurements for $R_{2}^{*}$ as well as for $S_{0}$ curves are summarized in Table 1 . The best overall CNR was achieved by the log-linear calculation according to Equation (5), followed by the 10 echo fit considering water and fat compartments. The CNR sum for the dual-echo correction was less precise with about $11 \%$ compared to the best value. In the normalized sum of Euclidean distances, the different methods appear in the same order, except for the calculation based on the first and second echoes only (see Table 1).

Most reliable pharmacokinetic parameters occurred to be $\mathrm{K}^{\mathrm{trans}}$ and $\mathrm{rBV}$. In Table 2 , their reproducibility is shown depending on different models (Tofts, Patlak or fit to a Gaussian or Gama Variate peak) and VIF selected for calculation.

\subsection{Vascular Input Function}

In general, the overall signal intensity in the slice aimed for the AIF estimation was significantly lower than in the tumor covering slice(s).

For all kinds of multi-echo correction, the CNR of the VOF was better than that of the AIF by a factor of 1.75 to 3.5, where the superior sagittal sinus allowed most plausible curves. For the preferred log-linear correction these factors amount to 2.96 for $S_{0}$ and 2.10 for $R_{2}^{*}$ resp. Perfusion evaluations using the VOF as vascular input function also result in the best reproducibility parameter $\bar{\Delta}$ (see next paragraph).

In addition, high reproducibility of perfusion parameters was achieved by using vascular input function from $R_{2}^{*}(t)$ within the tumor ROI.

\subsection{Comparison of Pharmacokinetic Model-Vascular Input Function Combinations}

The frequency of occurrence of the different models and vascular input functions in the list of the 30 best model-VIF combinations is summarized in Table 3. In this summary, we excluded the lacunarity parameters for reasons of plausibility (Apparently good lacunarity parameters mostly were based on parameter images calculated with bad performing VIFs. Hence, their information content probably consists on the tumor shape rather than on the inhomogeneity 
Table 2. Reproducibility parameter $\bar{\Delta}$ for $\mathrm{K}^{\text {trans }}$ (left) and rBV (right) from the 30 best performing combinations of models and VIFs. $K^{\text {trans }}$ (left) without normalization, rBV (right) after normalization to healthy tissue. Asterix indicates significant differences ( $\mathrm{p}<$ 0.05 , Student's t test).

\begin{tabular}{|c|c|c|c|c|c|c|c|c|}
\hline $\begin{array}{l}\mathrm{K}^{\text {trans }} \\
\text { model }\end{array}$ & $\begin{array}{l}\mathrm{rBV} \text { in } \\
\text { function }\end{array}$ & $\begin{array}{c}\text { VIF } \\
\text { source }\end{array}$ & $\begin{array}{l}\text { VIF } \\
\text { ROI }\end{array}$ & Z & $\begin{array}{l}\mathrm{rBV} \\
\text { model }\end{array}$ & $\begin{array}{c}\text { VIF } \\
\text { source }\end{array}$ & $\begin{array}{l}\text { VIF } \\
\text { ROI }\end{array}$ & $\bar{\Delta}$ \\
\hline Tofts & fitted & $R_{2}^{*}$ & tumor & 0.47 & Patlak & $S_{0}$ & vene & 0.61 \\
\hline Tofts & fix & $R_{2}^{*}$ & tumor & 0.51 & Gauss area & $R_{2}^{*}$ & vene & 0.71 \\
\hline Tofts & fitted & $R_{2}^{*}$ & vene & 0.58 & Gauss peak & $R_{2}^{*}$ & vene & 0.80 \\
\hline Tofts & fix & $R_{2}^{*}$ & vene & 0.76 & Patlak & $R_{2}^{*}$ & tumor & 0.82 \\
\hline Tofts & fitted & $R_{2}^{*}$ & artery & 0.81 & Tofts & $R_{2}^{*}$ & tumor & 0.85 \\
\hline Tofts & fitted & $S_{0}$ & vene & 1.16 & Tofts & $R_{2}^{*}$ & vene & 0.93 \\
\hline Tofts & fix & $R_{2}^{*}$ & artery & 1.17 & Patlak & $R_{2}^{*}$ & artery & 0.97 \\
\hline Patlak & fitted & $S_{0}$ & vene & 1.17 & Gauss area & $R_{2}^{*}$ & vene & 1.20 \\
\hline Patlak & fix & $R_{2}^{*}$ & tumor & 1.47 & Tofts & $S_{0}$ & vene & 1.28 \\
\hline Tofts & fix & $S_{0}$ & vene & 1.69 & Tofts & $R_{2}^{*}$ & artery & 1.43 \\
\hline Tofts & fix & $S_{0}$ & artery & 1.84 & GVF peak & $R_{2}^{*}$ & vene & 1.43 \\
\hline Tofts & fitted & $S_{0}$ & artery & 2.21 & Patlak & $R_{2}^{*}$ & vene & 1.47 \\
\hline Patlak & fix & $R_{2}^{*}$ & artery & 2.30 & Tofts & $S_{0}$ & artery & 2.97 \\
\hline Patlak & fitted & $R_{2}^{*}$ & vene & 2.60 & Patlak & $S_{0}$ & artery & 3.36 \\
\hline Patlak & fitted & $R_{2}^{*}$ & tumor & 2.68 & & & & \\
\hline Patlak & fitted & $R_{2}^{*}$ & artery & 2.87 & & & & \\
\hline Patlak & fix & $R_{2}^{*}$ & vene & 3.93 & & & & \\
\hline Patlak & fix & $S_{0}$ & vene & 4.02 & & & & \\
\hline Patlak & fitted & $S_{0}$ & artery & 4.41 & & & & \\
\hline Patlak & fix & $S_{0}$ & artery & 4.67 & & & & \\
\hline
\end{tabular}

Table 3. Percentage of models and vascular input functions in the list of 30 best parameters relative to the number of their occurrence in the list of all calculations performed. Middle column: plain parameters. Right column: parameters normalized to healthy tissue. Fractal parameters are not included. The type “---" refers to model free simple parameters (like maximum enhancement e.g.) or parameters which were calculated independent of a vascular input function resp.

\begin{tabular}{lcc}
\hline Model & Non normalized & Normalized \\
\hline Tofts & $7.1 \%$ & $17.1 \%$ \\
Patlak & $0.0 \%$ & $13.3 \%$ \\
IDT & $0.0 \%$ & $0.0 \%$ \\
Gaussian fit & $0.0 \%$ & $50.0 \%$ \\
GVF fit & $0.0 \%$ & $0.0 \%$ \\
--- & $25.0 \%$ & $50.0 \%$ \\
Vascular input function & Non normalized & Normalized \\
$S_{0}$ artery & $0.0 \%$ & $20.0 \%$ \\
$S_{0}$ vein & $0.0 \%$ & $11.1 \%$ \\
$R_{2}^{*}$ artery & $0.0 \%$ & $0.0 \%$ \\
$R_{2}^{*}$ vein & $4.3 \%$ & $21.7 \%$ \\
$R_{2}^{*}$ tissue & $15.0 \%$ & $30.0 \%$ \\
--- & $14.3 \%$ & $21.4 \%$ \\
\hline
\end{tabular}


properties). The most reliable calculations are based on the Extended Toftsmodel, Gaussian fit and model-free calculations (like maximum enhancement e.g., which showed minimum $\bar{\Delta}$ of all parameters normalized to healthy tissue).

The results of IDT calculations are rather dissatisfactory. None of the IDTbased parameters was found either in the 30 best normalized or the best nonnormalized parameters.

\subsection{Signal Decomposition}

The lowest mean "fat-to-water" signal ratio was found for malignant glioma and the highest mean for meningioma. Besides this, higher "fat-to-water" signal ratios were detected in gliomas after dexamethasone application, compared to those without such medication. But due to the small sample size, these differences were not statistically significant.

\section{Discussion}

Dual-echo perfusion measurements have already been proposed in the 1990s. They allow calculation of the time course of $R_{2}^{*}(t)$ and extrapolation of the signal intensity to an echo time $T_{E}=0$. Thus, $T_{1}$ and $T_{2}^{*}$ related effects can be separated into independent functions of time. However, the clinical use of this principle is limited by the small number of slices which can be examined with sufficient temporal resolution. This disadvantage could be overcome by different schemes of $k$-space undersampling or segmented EPI [e.g. [5] [18] [19] [20] [21]].

Compared to the dual-echo approach, the multi-echo approach that is proposed in this paper allows improvements concerning signal-to-noise ratio and estimation of vascular input functions. Furthermore, it allows at least rough estimations of the proportion of components with different Larmor frequencies.

\subsection{Comparison of Multi-Echo Correction Methods (Calculation of $\boldsymbol{R}_{2}^{*}$ and $\mathrm{S}_{0}$ )}

In our study, the loglinear curve fit was found to suit best among all multi-echo correction algorithms tested. Remarkable, loglinear correction showed better results than the exponential one. From the physical point of view, however, the exponential fits correspond to the data more exactly than fits on logarithmized signal intensities (by taking the logarithms, the statistical uncertainty of higher values is compressed. Thus, linear fitting of logarithms overweighs the low-intensity data points corresponding to the late echoes). Otherwise, for numerical reasons, they are less stable compared to the linear fit on the logarithmized data. In other words, if the weighting of data points influences the curve fit to an important way, then the exponential fits should give better results than the loglinear ones. But, if numerical robustness is the essential objective of the multiecho correction method, then the loglinear model should work better than the exponential ones. Therefore, in our data the stability aspect underwent more influence than the biased weighting of the data points. 
We also found the biexponential fat-water models resulting in higher Euclidean distances between tumor entities and $\mathrm{CNR}$-at least compared to the corresponding monoexponential models. But we want to stress, that models based on loglinear and monoexponential calculations are actually identical, the only differences are the different weighting of data points and the different numeric stability.

An extended discussion of the biexponential fat-water model can be found in the "signal decomposition" paragraph.

Testing simple dual-echo corrections, Euclidean distances between tumor entities based on $\mathrm{K}^{\text {trans }}$ and $\mathrm{rBV}$, appeared to be surprisingly good for the first and second echo only - in contrast to the CNR evaluation. This, however, seems to be a statistical artifact due to the high data noise and the small sample size. Obtained parameters were not significantly biased compared to the ones from the other correction methods, but they were noisier.

\subsection{Vascular Input Function}

A prerequisite for quantitative perfusion measurements is the determination of VIF. Several approaches of manual, semi-automatic or automatic VIF determinations for brain perfusion measurements are available. There are for example measurements of a special slice caudal to the tumor for deriving of an AIF [22], automatic selection of pixels within the brain with highest and/or fastest enhancement [23] or with clustering algorithms [24], reference to the venous output function [25] or VIF estimation from averaging over several patients [13] [26].

In the present study, we drew the ROIs manually in order to control their influence on the time courses of $S_{0}$ and $R_{2}^{*}$. In this way, we could avoid possible underestimation of peak changes due to partial volume and non-plausible curve shapes resulting from flow effects. It should be noted here, that the definition of an arterial ROI often was challenging. In some cases it was even not possible to obtain plausible time courses of $S_{0}$ and/or $R_{2}^{*}$-possibly due to higher influence of turbulent flow on signal intensity at time with higher CM concentration. Another cause for implausible AIF curves in some cases was the bias of the magnitude of the signal intensity due to noise, especially for the late echoes leading to underestimation of $R_{2}^{*}$ during the bolus peak.

In our data, nonlinearity of $S_{0}$ (CM concentration) as well as biased curve shapes in the venous ROIs were less noticeable than in the arterial ones. This subjective impression was confirmed by CNR analysis as well as by rating of perfusion parameters with different vascular input functions. According to Yuan [25], the advantage of venous time courses can be explained by lower flow velocity as well as more steady flow in veins compared to arteries. Additionally, in their work, VIFs derived from veins occurred to be less dependent on the selected slice than that derived from arteries.

Earlier studies [27] [28] demonstrated the crucial influence of the temporal shift of vascular input function on the estimation of perfusion parameters. Ap- 
plying multi-echo sequences, this problem can be avoided by the measurement of $R_{2}^{*}(t)$ in the tumor ROI. The peak position of $R_{2}^{*}(t)$ can then be used for optimal time shift of a vascular input function measured elsewhere.

In addition to the correct position on the time axis, $R_{2}^{*}(t)$ from the tumor ROI possesses the correct curve dispersion or peak broadening. This could be the reason for the quite good stability of perfusion parameters calculated with this VIF. These properties even overcompensates the worse signal-to-noise ratio compared to the vessel-based input functions.

However, due to downscaling by the volume fraction of capillaries in the tumor voxels, $R_{2}^{*}(t)$ from the tumor ROI as vascular input function is nevertheless acceptable concerning position and peak width, but is not acceptable concerning the amplitude. Hence, perfusion parameters determined this way have to be corrected for this downscaling. ${ }^{1}$

\subsection{Comparison of Pharmacokinetic Model-Vascular Input Function Combinations}

Although models with fewer numbers of free parameters like the Patlak approach are supposed to be more stable, we found better reproducibility with the Tofts model. This is a contradictory finding to previously published data [26] [29]. In [30], owing to more fitting parameters, the two-compartment exchange model occurred to give less stable results at given acquisition duration compared to the Tofts and Extended Tofts models as well. The better reproducibility found in our data applying the Extended Tofts model might be explained by the fact, that in contrary to the Extended Tofts, in Patlak model one or two boundaries on the time axis (depending on the implementation) have to be chosen. The uncertainty during manual selection of these boundaries might overcompensate the a-priori better stability of fits to model functions with fewer degrees of freedom. It has to be evaluated, to what degree automatic determination of the upper (later) boundary based on statistical methods could reduce this uncertainty.

Calculations based on the Indicator Dilution Theory lead to quite instable results. This could be caused by the limited influence of smaller, even good vascularized tumors on the Mean Transit Time (MTT) of the CM bolus due to the comparatively short pathway of the blood through the tumor. Another reason could be the less stable rBV estimation by means of the fit of a Gamma Variate Function compared to the well performing Gaussian fit.

Diagnostics based on pharmacological parameters is one way to achieve comparability of results between different sites. Another way to reach this aim is standardization of measurement conditions allowing for comparison of simple model free parameters. In MR mammography, a qualitative description of the time course of contrast enhancement under mildly standardized measurement conditions occurred to meet the clinical needs [31]. In our data, among all parameters normalized to healthy tissue, the best reproducibility was obtained for

${ }^{1}$ The formal parameter " $r B V$ " as well as $\mathrm{K}^{\text {trans }}$ in the Extended Tofts and Patlak models have to be divided by rBV - meaning either extracting the root of the fit parameter "rBV" or applying a rBV determined by another vascular input function (e.g. the venous $R_{2}^{*}(t)$ ). 
the signal increase extrapolated to $\mathrm{TE}=0$, which is influenced by vessel permeability, perfusion and extravascular extracellular space.

\subsection{Signal Decomposition}

The assumption for brain and tumor tissue to consist of two components with different Larmor frequency appears to improve the quality of curve fits of signal intensities such as a function of TE, compared to monoexponential fits without differentiation between fat and water signals. This is clearly seen in the evaluation of CNRs, but does not transfer to better accuracy of perfusion parameter based differentiation between tumor entities (Table 1). However, we demonstrated decreased lower-frequency-compartment (with a chemical shift in the range of fatty tissue) in gliomas after pretreating with dexamethasone compared to the untreated group (Table 4, Figure 2). Thus, the multi-echo perfusion approach has the potential to provide additional physiological information beyond the description of blood supply. The background of these findings has to be analyzed comparing such measurements with in-vivo MR spectroscopy.

\subsection{Limitations}

Some limitations of this study are to be mentioned:

First of all, our data was not compared to any reference method. To our knowledge, no real "gold standard" for the perfusion measures exists. Hence, for the evaluation of the reliability of our data, we introduced the reproducibility parameter defined in Equation (1).

Second, evaluations were performed with in-house software. Although carefully tested during the development process, there remains a higher risk of incorrect calculations or numeric instabilities compared to commercial and/or widespread software solutions. No reference calculations with standard software were performed.

Finally, in the literature, DCE MR studies of brain tumors usually are performed for about 5 minutes at a temporal resolution of $5.3 \mathrm{~s}$ [32] resulting in about 55 time points. In our study, we needed a temporal resolution of 2 seconds or higher for the DSC evaluations. At the same time, this denser sampling of 60 time points within two minutes should at least partly compensate the lack of very late data points needed for determination of $K^{\text {trans }}$ and $v_{e}$ or $k_{2}$ resp.

\section{Summary}

The extension of the well-known dual-echo perfusion measurement to multiecho sequence leads to the advantage of improved signal-to-noise ratios. The most reliable determinations of perfusion parameters (especially $\mathrm{K}^{\text {trans }}$ and $\mathrm{rBV}$ ) were achieved with Extended Tofts model applying the $R_{2}^{*}$ related venous output function or output function estimated in the tumor tissue.

Temporal distribution of contrast concentration within the tumor vessel bed from multi-echo data seems to be advantageous for the estimation of a vascular 
Table 4. Mean values of fat to water signal ratios for glioma without vs. with pretreatment with dexamethasone.

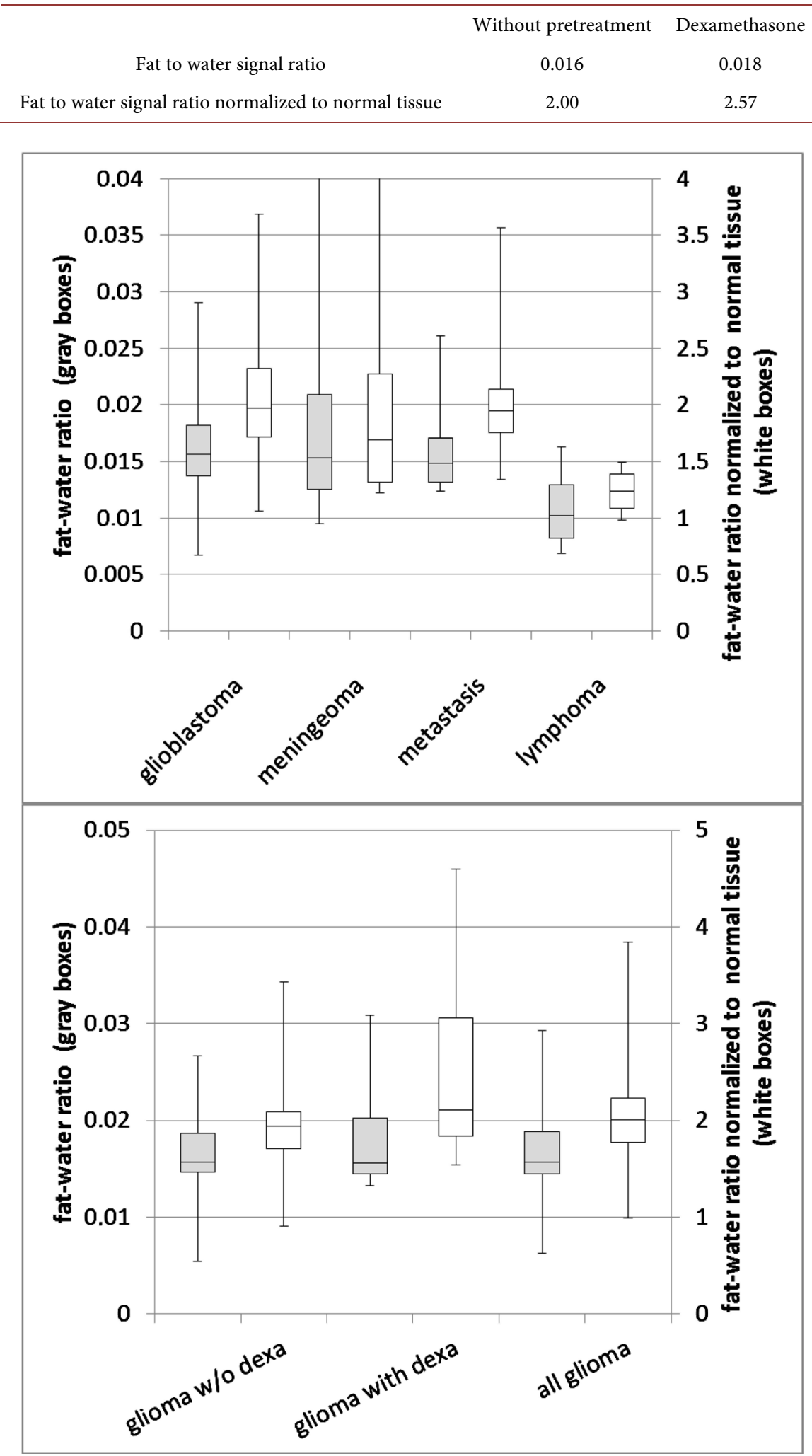

Figure 2. Box plot for fat-water ratios estimated with Equation (8).

input function compared to potentially dispersed and time-shifted functions de- 
rived from arterial or venous signals.

As potential added value, multi-echo FLASH MRI allowing signal decomposition into two components with different Larmor frequencies might provide additional information concerning tissue composition of brain tumors. The clinical value of these findings and particularly the exact biochemical composition of the so-called "fat" component are fruitful areas for of future research.

\section{Acknowledgements}

We gratefully thank Nadine Hietschold for her helpful recommendations and linguistic support during the writing of this manuscript.

\section{References}

[1] Rosen, B.R., Belliveau, J.W., Vevea, J.M. and Brady, T.J. (1990) Perfusion Imaging with NMR Contrast Agents. Magnetic Resonance in Medicine, 14, 249-265. https://doi.org/10.1002/mrm.1910140211

[2] Hietschold, V. (2004) Aspekte einer quantitativen Gewebecharakterisierung mittels Kontrastmitteldynamik in der Kernspintomographie. Cuvillier, Göttingen.

[3] El Khouli, R.H., Macura, K.J., Barker, P.B., Habba, M.R., Jacobs, M.A. and Bluemke, D.A. (2009) Relationship of Temporal Resolution to Diagnostic Performance for Dynamic Contrast Enhanced MRI of the Breast. Journal of Magnetic Resonance Imaging, 30, 999-1004. https://doi.org/10.1002/jmri.21947

[4] Gutierrez, R.L., Strigel, R.M., Partridge, S.C., DeMartini, W.B., Eby, P.R., Stone, K.M., Peacock, S. and Lehman, C.D. (2012) Dynamic Breast MRI: Does Lower Temporal Resolution Negatively Affect Clinical Kinetic Analysis? American Journal of Roentgenology, 199, 703-708. https://doi.org/10.2214/AJR.11.7836

[5] Grøvik, E., Bjørnerud, A., Storås, T.H. and Gjesdal, K.I. (2014) Split Dynamic MRI: Single Bolus High Spatial-Temporal Resolution and Multi Contrast Evaluation of Breast Lesions. Journal of Magnetic Resonance Imaging, 39, 673-682. https://doi.org/10.1002/jmri.24206

[6] Miyati, T., Banno, T., Mase, M., Kasai, H., Shundo, H., Imazawa, M. and Ohba, S. (1997) Dual Dynamic Contrast-Enhanced MR Imaging. Journal of Magnetic Resonance Imaging, 7, 230-235. https://doi.org/10.1002/jmri.1880070136

[7] Hietschold, V., Klengel, S. and Köhler, K. (1993) Simultaneous Dynamic Measurement of Tissue Contrast Enhancement and Perfusion at 0.5 Tesla: Method and Postprocessing. SMRM 11th Annual Meeting, New York, 16-20 August 1993, Book of Abstracts 619.

[8] Heiland, S., Benner, T., Rempp, K., Reith, W., Forsting, M. and Sartor, K. (1997) Ein Verfahren zur simultanen Beurteilung der Hämodynamik und der dynamischen Kontrastmittelanreicherung bei zerebralen Läsionen mit gestörter Blut-HirnSchranke. Fortschr Röntgenstr, 166, S137.

[9] Calamante, F. (2013) Arterial Input Function in Perfusion MRI: A Comprehensive Review. Progress in Nuclear Magnetic Resonance Spectroscopy, 74, 1-32. https://doi.org/10.1016/j.pnmrs.2013.04.002

[10] Patlak, C.S., Blasberg, R.G. and Fenstermacher, J.D. (1983) Graphical Evaluation of Blood-to-Brain Transfer Constants from Multiple-Time Uptake Data. Journal of Cerebral Blood Flow and Metabolism, 3, 1-7. https://doi.org/10.1038/jcbfm.1983.1

[11] Tofts, P.S. (1997) Modeling Tracer Kinetics in Dynamic Gd-DTPA Imaging. JMR, 7, 91-101. https://doi.org/10.1002/jmri.1880070113 
[12] Tofts, P.S., Brix, G., Buckley, D.L., Evelhoch, J.L., Henderson, E., Knopp, M.V., Larsson, H.B.W., Lee, T.Y., Mayr, N.A., Parker, G.J.M., Port, R.E., Taylor, J. and Weisskoff, R.M. (1999) Estimating Kinetic Parameters From Dynamic ContrastEnhanced $\mathrm{T}_{1}$-Weighted MRI of a Diffusable Tracer: Standardized Quantities and Sym-bols. Journal of Magnetic Resonance Imaging, 10, 223-232.

https://doi.org/10.1002/(SICI)1522-2586(199909)10:3<223::AID-JMRI2>3.0.CO;2-S

[13] Bergamino, M., Bonzano, L., Levrero, F., Mancardi, G.L. and Roccatagliata, L. (2014) A Review of Technical Aspects of $\mathrm{T}_{1}$-Weighted Dynamic Contrast-Enhanced Magnetic Resonance Imaging (DCE-MRI) in Human Brain Tumors. Physica Medica, 30, 635-643. https://doi.org/10.1016/j.ejmp.2014.04.005

[14] Meier, P. and Zierler, K.L. (1954) On the Theory of the Indicator-Dilution Method for Measurement of Blood Flow and Volume. Journal of Applied Physiology, 6, 731744.

[15] Zierler, K.L. (1962) Theoretical Basis of Indicator-Dilution Methods for Measuring Flow and Volume. Circulation Research, 10, 393-407. https://doi.org/10.1161/01.RES.10.3.393

[16] Clough, A.V., Manuel, A.J., Haworth, S.T. and Dawson, C.A. (2000) Application of Indicator Dilution Theory to Time-Density Curves Obtained from Dynamic Contrast Images. Proc. SPIE 3978, Medical Imaging 2000: Physiology and Function from Multidimensional Images, Location, 20 April 2000, 457.

[17] Abramyuk, A., Hietschold, V., Appold, S., von Kummer, R. and Abolmaali, N. (2015) Radiochemotherapy-Induced Changes of Tumour Vascularity and Blood Supply Estimated by Dynamic Contrast-Enhanced CT and Fractal Analysis in Malignant Head and Neck Tumours. The British Journal of Radiology, 88, 20140412. https://doi.org/10.1259/bjr.20140412

[18] Yoruk, U., Saranathan, M., Loening, A.M., Hargreaves, B.A. and Vasanawa, S.S. (2016) High Temporal Resolution Dynamic MRI and Arterial Input Function for Assessment of GFR in Pediatric Subjects. Magnetic Resonance in Medicine, 75, 1301-1311. https://doi.org/10.1002/mrm.25731

[19] Jelescu, I.O., Leppert, I.R., Narayanan, S., Araújo, D., Arnold, D.L. and Pike, G.B. (2011) Dual-Temporal Resolution Dynamic Contrast-Enhanced MRI Protocol for Blood-Brain Barrier Permeability Measurement in Enhancing Multiple Sclerosis Lesions. Journal of Magnetic Resonance Imaging, 33, 1291-1300. https://doi.org/10.1002/jmri.22565

[20] Pierre, E.Y., Ma, D., Chen, Y., Badve, C. and Griswold, M.A. (2016) Multiscale Reconstruction for MR Fingerprinting. Magnetic Resonance in Medicine, 75, 2481 2492. https://doi.org/10.1002/mrm.25776

[21] Stokes, A.M. and Quarles, C.C. (2016) A Simplified Spin and Gradient Echo Approach for Brain Tumor Perfusion Imaging. Magnetic Resonance in Medicine, 75, 356-362. https://doi.org/10.1002/mrm.25591

[22] Rijpkema, M., Kaanders, J.H.A.M., Joosten, F.B.M., van der Kogel, A.J. and Heerschap, A. (2001) Method for Quantitative Mapping of Dynamic MRI Contrast Agent Uptake in Human Tumors. JMRI, 14, 457-463.

https://doi.org/10.1002/jmri.1207

[23] Chen, J., Yao, J. and Thomasson, D. (2008) Automatic Determination of Arterial Input Function for Dynamic Contrast Enhanced MRI in Tumor Assessment. $\mathrm{Me}$ dical Image Computing and Computer-Assisted Intervention, 11, 594-601. https://doi.org/10.1007/978-3-540-85988-8_71

[24] Yin, J., Yang, J. and Guo, Q. (2015) Automatic Determination of the Arterial Input Function in Dynamic Susceptibility Contrast MRI: Comparison of Different Re- 
producible Clustering Algorithms. Neuroradiology, 57, 535-543. https://doi.org/10.1007/s00234-015-1493-9

[25] Yuan, J., Chow, S.K.K., Zhang, Q., Yeung, D.K.W., Ahuja, A.T. and King, A.D. (2013) The Use of Dynamic Tracer Concentration in Veins for Quantitative DCEMRI Kinetic Analysis in Head and Neck. PLOS ONE, 8, e59885. https://doi.org/10.1371/journal.pone.0059885

[26] Shukla-Dave, A., Lee, N., Stambuk, H., Wang, Y., Huang, W., Thaler, H.T., Patel, S.G., Shah, J.P. and Koutcher, J.A. (2009) Average Arterial Input Function for Quantitative Dynamic Contrast Enhanced Magnetic Resonance Imaging of Neck Nodal Metastases. BMC Medical Physics, 9, 4. https://doi.org/10.1186/1756-6649-9-4

[27] Natsume, T., Ishida, M., Kitagawa, K., Nagata, M., Sakuma, H. and Ichihara, T. (2015) Theoretical Considerations in Measurement of Time Discrepancies between Input and Myocardial Time-Signal Intensity Curves in Estimates of Regional Myocardial Perfusion with First-Pass Contrast-Enhanced MRI. Magnetic Resonance in Medicine, 33, 1059-1065. https://doi.org/10.1016/j.mri.2015.06.015

[28] Hietschold, V., Gohl, P., Abramyuk, A., Koch, A., Laniado, M. and Abolmaali, N. (2007) Evaluation of CT Perfusion Results from Human Tumors: A Comparison of Four Different Two-Compartment Models. Radiological Society of North America, Scientific Assembly and Annual Meeting, Chicago, 25 November-30 November 2007. http://archive.rsna.org/2007/5003088.html

[29] Gaens, M.E., Backes, W.H., Rozel, S., Lipperts, M., Sanders, S.N., Jaspers, K., Cleutjens, J.P.M., Sluimer, J.C., Heeneman, S., Daemen, M.J.A.P., Welten, R.J.T.J., Daemen, J.W.H., Wildberger, J.E., Kwee, R.M. and Kooi, M.E. (2013) Dynamic Contrast-Enhanced MR Imaging of Carotid Atherosclerotic Plaque: Model Selection, Reproducibility, and Validation. Radiology, 266, 271-279.

https://doi.org/10.1148/radiol.12120499

[30] Hindel, S., Söhner, A., Maaß, M., Sauerwein, W., Baba, H.A., Kramer, M. and Lüdemann, L. (2017) Validation of Interstitial Fractional Volume Quantification by Using Dynamic Contrast-Enhanced Magnetic Resonance Imaging in Porcine Skeletal Muscles. Investigative Radiology, 52, 66-73. https://doi.org/10.1097/RLI.0000000000000309

[31] Neubauer, H., Li, M., Kuehne-Heid, R., Schneider, A. and Kaiser, W.A. (2003) High Grade and Non-High Grade Ductal Carcinoma in Situ on Dynamic MR Mammography: Characteristic Findings for Signal Increase and Morphological Pattern of Enhancement. The British Journal of Radiology, 76, 3-12.

https://doi.org/10.1259/bjr/14883856

[32] Heye, A.K., Culling, R.D., Valdez Hernandez, M., Trippleton, M.J. and Wardlaw, J.M. (2014) Assessment of Blood-Brain Barrier Disruption Using Dynamic Contrast-Enhanced MRI. A Systematic Review. NeuroImage: Clinical, 6, 262-274. https://doi.org/10.1016/j.nicl.2014.09.002

[33] Wang, H.Z., Riederer, S.J. and Lee, J.N. (1987) Optimizing the Precision in T1 Relaxation Estimation Using Limited Flip Angles. Magnetic Resonance in Medicine, 5, 399-416. https://doi.org/10.1002/mrm.1910050502

[34] Dixon, W.T. (1984) Simple Proton Spectroscopic Imaging. Radiology, 153, 189-194. https://doi.org/10.1148/radiology.153.1.6089263

[35] Glover, G.H. (1991) Multipoint Dixon Technique for Water and Fat Proton and Susceptibility Imaging. Journal of Magnetic Resonance Imaging, 1, 521-530. https://doi.org/10.1002/jmri.1880010504

[36] Ma, J. (2008) Dixon Techniques for Water and Fat Imaging. JMRI, 28, 543-558. 
https://doi.org/10.1002/jmri.21492

[37] Hernando, D., Kramer, J.H. and Reeder, S.B. (2013) Multipeak Fat-Corrected Complex R2* Relaxometry: Theory, Optimization, and Clinical Validation. Magnetic Resonance in Medicine, 70, 1319-1331. https://doi.org/10.1002/mrm.24593 


\section{Appendix: Calculation of $R_{2}^{*}(t)$ and $S_{0}(t)$}

According to Wang et al. [33], for gradient echo sequences the signal intensity is given as

$$
S=\rho \cdot \frac{\exp \left(\frac{-T_{E}}{T_{2}^{*}}\right) \cdot \sin (\alpha) \cdot\left(1-\exp \left(\frac{-T_{R}}{T_{1}}\right)\right)}{1-\exp \left(\frac{-T_{R}}{T_{1}}\right) \cdot \exp \left(\frac{-T_{R}}{T_{2}^{*}}\right)-\cos (\alpha) \cdot\left(\exp \left(\frac{-T_{R}}{T_{1}}\right)-\exp \left(\frac{-T_{R}}{T_{2}^{*}}\right)\right)}
$$

For $\mathrm{T}_{2}^{*} \ll \mathrm{TR}$ it simplifies to the commonly used form

$$
S=\rho \cdot \sin (\alpha) \cdot \exp \left(\frac{-T_{E}}{T_{2}^{*}}\right) \frac{1-\exp \left(\frac{-T_{R}}{T_{1}}\right)}{1-\cos (\alpha) \cdot \exp \left(\frac{-T_{R}}{T_{1}}\right)}
$$

In principle, this simplification condition is not fulfilled for all data points in our experiments. However, in the relevant ranges of $T_{1}$ and $T_{2}^{*}$, the influence of $T_{R} / T_{2}^{*}$ is small compared to the nonlinear dependence of $S$ on $T_{1}$. In addition, using Equation (2) requires the knowledge of native $T_{1}$ values. To our experience, the determination of $T_{1}$ introduces uncertainties, which could even prevail the possible advantage of the elimination of this nonlinearity. In addition, the separation of the influences of $T_{1}$ and $T_{2}^{*}$ into distinct factors in Equation (3) is of great numerical advantage. Hence, the multi-echo data were converted to $R_{2}^{*}$ and $S_{0}$ maps based on Equation (3) ( $S_{0}$ is the hypothetical signal intensity for $T_{E}$ $=0$, i.e., the signal part which depends on proton density and $T_{1}$ only) either by logarithmized ratios of two signal intensities

$$
\begin{gathered}
R_{2}^{*}=\ln \left(S_{0} / S_{i}\right) /\left(T_{E, i}-T_{E, 0}\right) \\
S_{0}=S_{1} \cdot \exp \left(T_{E, 1} \cdot R_{2}^{*}\right)
\end{gathered}
$$

with $i=[1,2]$ or $i=[1,9]$ (smallest or largest difference in echo times), by linear fit of 10 logarithmic signal intensities

$$
\ln \left(S_{i}\right)=R_{2}^{*} \cdot T_{E, i}+f\left(T_{1}\right)
$$

with $i=0, \cdots, 9$ or by a monoexponential fit

$$
S_{i}=\exp \left(-T_{E, i} \cdot R_{2}^{*}\right) \cdot f\left(T_{1}\right)
$$

with four or all ten echoes $(i=[0,1,4,9]$ or $i=[0, \cdots, 9])$.

In addition, according to Dixon [34] [35] [36] a signal decomposition to two components with different Larmor frequency (here called "water" and "fat" signals $\left(W\left(T_{1}\right)\right.$ and $F\left(T_{1}\right)$ resp.) based on the phase differences for these components for every echo time was performed

$$
S_{i}=\exp \left(-T_{E, i} \cdot R_{2}^{*}\right) \cdot \sqrt{\left(W+F \cos \left(\Delta \alpha_{i}\right)\right)^{2}+\left(F \sin \left(\Delta \alpha_{i}\right)\right)^{2}}
$$

$(i=[0,1,4,9]$ or $i=[0, \cdots, 9])$ with

$$
\Delta \alpha_{i}=T_{E, i} \cdot 2 \pi \cdot \Delta f
$$


( $\Delta f$ - frequency difference for the water vs. fat spins which is given by the water Larmorfrequency times the fat-water chemical shift). From Equation (8) (which is the adaption of the formula given in [35] to real numbers eliminating the influence of additional phase shifts with only slight noise penalty [37]), a fat to water signal ratio $F / W$ can be derived.

In the selection of the four echoes for Equations ((7) and (8)) a more or less equal signal difference between consecutive echoes for characteristic $T_{2}^{*}$ values was intended.

In the dependence of the signal intensity on TE, noise was neglected even for long $T_{E}$ and high CM concentration. In previous evaluations we could show, that noise was small compared to the signal, even at peak $R_{2}^{*}$ for the longest $T_{E}$ for all regions of interest except for some cases with very small ROI for the AIF and poor signal intensity in the AIF slice. But even in these cases, the noise-related signal at long $T_{E}$ was small compared to nonstochastic deviations of $S\left(T_{E}\right)$ from a monoexponential form.

Submit or recommend next manuscript to SCIRP and we will provide best service for you:

Accepting pre-submission inquiries through Email, Facebook, LinkedIn, Twitter, etc. A wide selection of journals (inclusive of 9 subjects, more than 200 journals)

Providing 24-hour high-quality service

User-friendly online submission system

Fair and swift peer-review system

Efficient typesetting and proofreading procedure

Display of the result of downloads and visits, as well as the number of cited articles

Maximum dissemination of your research work

Submit your manuscript at: http://papersubmission.scirp.org/

Or contact ijmpcero@scirp.org 